ARTICLE

Received 9 Nov 2014 | Accepted 6 May 2015 | Published 15 Jun 2015 DOl: 10.1038/ncomms8404

\title{
Defective Hfp-dependent transcriptional repression of $d M Y C$ is fundamental to tissue overgrowth in Drosophila XPB models
}

Jue Er Amanda Lee ${ }^{1, \star}$, Naomi C. Mitchell ${ }^{1} \star$, Olga Zaytseva ${ }^{1}$, Arjun Chahal ${ }^{1}$, Peter Mendis ${ }^{1}$, Amandine Cartier-Michaud ${ }^{2, \dagger}$, Linda M. Parsons ${ }^{1}$, Gretchen Poortinga ${ }^{3}$, David L. Levens ${ }^{4}$, Ross D. Hannan ${ }^{3,5}$ \& Leonie M. Quinn ${ }^{1}$

Nucleotide excision DNA repair (NER) pathway mutations cause neurodegenerative and progeroid disorders (xeroderma pigmentosum (XP), Cockayne syndrome (CS) and trichothiodystrophy (TTD)), which are inexplicably associated with (XP) or without (CS/TTD) cancer. Moreover, cancer progression occurs in certain patients, but not others, with similar C-terminal mutations in the XPB helicase subunit of transcription and NER factor $\mathrm{TFIIH}$. Mechanisms driving overproliferation and, therefore, cancer associated with XPB mutations are currently unknown. Here using Drosophila models, we provide evidence that C-terminally truncated Hay/XPB alleles enhance overgrowth dependent on reduced abundance of RNA recognition motif protein $\mathrm{Hfp} / \mathrm{FIR}$, which transcriptionally represses the MYC oncogene homologue, $d M Y C$. The data demonstrate that $d M Y C$ repression and dMYC-dependent overgrowth in the Hfp hypomorph is further impaired in the C-terminal Hay/XPB mutant background. Thus, we predict defective transcriptional repression of MYC by the $\mathrm{Hfp}$ orthologue, FIR, might provide one mechanism for cancer progression in $\mathrm{XP} / \mathrm{CS}$.

\footnotetext{
${ }^{1}$ Department of Anatomy and Neuroscience, University of Melbourne, Parkville, Melbourne 3010, Australia. ${ }^{2}$ Aix-Marseille University, F-13284 Marseille, France. ${ }^{3}$ Peter MacCallum Cancer Centre, St Andrews Place, East Melbourne Victoria 3002, Australia. ${ }^{4}$ Center for Cancer Research, National Cancer Institute, NIH, Bethesda, Maryland 20892, USA. ${ }^{5}$ Department of Cancer Biology and Therapeutics, The John Curtin School of Medical Research, The Australian National University, Canberra Australian Capital Territory 2600, Australia. ${ }^{\star}$ These authors contributed equally to this work. $\dagger$ Present address: Centre de Recherche en Cancérologie de Marseille, Inserm, U1068; Institut Paoli-Calmettes; CNRS, UMR7258, Marseille F-13009, France. Correspondence and requests for materials should be addressed to L.M.Q. (email: I.quinn@unimelb.edu.au).
} 
F or cell cycle patterning of multicellular animals, cells must have the capacity to quickly respond to extracellular developmental signals. Recent work has highlighted the importance of poised (transcriptionally engaged, but paused) RNA Polymerase II (RNA Pol II) on the promoters of Drosophila embryonic patterning genes as a mechanism to facilitate rapid and synchronous transcription in response to secreted morphogens ${ }^{1-7}$. Preceding this interest in RNA Pol II pausing, earlier ex vivo studies identified poised RNA Pol II at the transcriptional start site (TSS) of the MYC oncogene ${ }^{8-10}$. MYC drives cell growth and cell cycle progression ${ }^{3,11}$ and is frequently dysregulated in cancer ${ }^{12-15}$. $d M Y C$ is the sole MYC family member in Drosophila ${ }^{16-18}$ and is functionally homologous to $M Y C^{16,19-22}$. As even subtle increases in MYC expression (more than twofold) can promote cancer initiation and progression, MYC abundance must be tightly regulated ${ }^{14-16,23,24}$. Due to the short half life and low steady-state abundance of both $M Y C$ mRNA and protein, multiple mechanisms operate to constrain MYC (for example, transcription, mRNA turnover, translation and protein degradation) $)^{16,23,25-32}$. Ex vivo studies suggest paused, but transcriptionally engaged, RNA Pol II at the start site of the MYC promoter provides (1) rapid response to mitogenic signals and (2) protection from unwanted promoter activation $^{16,33,34}$.

Activation of RNA Pol II is dependent on general transcription factor IIH $(\mathrm{TFIIH})^{35,36}$, a multi-subunit complex essential for nucleotide excision repair (NER) and RNA Pol II-dependent transcription $7,16,23,24$. The complex genetic disorders xeroderma pigmentosum (XP), Cockayne syndrome (CS), trichothiodystrophy (TTD) and combined XP/CS are caused by mutations in NER genes, including XPB (encoded by ERCC3) the helicase subunit of TFIIH ${ }^{16,24,37-39}$. In contrast to CS and TTD, which are not associated with cancer, XP patients are extremely photosensitive and have a 1,000-fold increased risk of skin cancer, and 10-to-20 times higher risk of internal cancers $1,3,5,7,40$. However, despite years of study investigating the NER pathway in this group of diseases the outstanding question remains-why do certain mutations result in cancer phenotypes in some patients, but not others?

Although $>28$ NER proteins have been implicated in XP, TTD, CS and XP/CS, other than XPB, only the XPD helicase and TTDA are TFIIH subunits. TTDA is required for the stability of the TFIIH complex, but unlike XPB and XPD, which are implicated in all three diseases, defects in TTDA only cause the developmental disease TTD, not XP/CS and/or cancer ${ }^{8,41}$. Intriguingly, three different XPB families with mutations in ERCC3, the gene coding XPB, causing loss of the wild-type $\mathrm{C}$ terminus of XPB, all display the severe neurodegenerative progeroid disorder characteristic of CS, but have different cancer predisposition (one severe, one moderate, one with no cancer $)^{3,41,42}$. Altogether with the knowledge that all patients in these families have reduced NER, these observations suggest that cancer phenotypes are not due to NER defects per se, but likely due to a second mutation. Thus we predict that XPB-dependent DNA repair defects are not the direct cause of cancer phenotypes, but are a consequence of additional hyperproliferative input(s), perhaps involving the transcription of a gene driving tissue overgrowth.

Serum stimulation of mammalian tissue culture cells results in a rapid increase in $M Y C$ transcription ${ }^{12,43}$, which is associated with release of RNA Pol II from the TSS ${ }^{16,44}$. Subsequently, RNA Pol II is restored to the TSS and MYC transcription is returned to basal levels, consistent with the poised RNA Pol II complex at the $M Y C$ TSS being required to shut down $M Y C^{16,43}$. Indeed at other gene promoters, paused RNA Pol II appears to govern inappropriate transcription, presumably as a consequence of steric hindrance from the multi-subunit RNA Pol II complex blocking stochastic recruitment of RNA Pol II ${ }^{20,22,44}$.

XPB regulates post-initiation control of $M Y C$ transcription via the RNA recognition motif protein FIR and the $\mathrm{KH}$ domain protein $\mathrm{FBP}^{16,23,24,45}$. Specifically, under the conditions of activated (serum stimulated) MYC transcription, XPB is required for recruiting both FBP and FIR to a single-stranded promoter sequence (the far upstream sequence element) $1.7 \mathrm{kB}$ upstream of the MYC TSS ${ }^{16,23,40}$. FIR is recruited subsequent to the peak in $M Y C$, is proposed to inactivate RNA Pol II and is required to return $M Y C$ transcription to basal levels ${ }^{16,41}$. Indeed, loss-of-function FIR mutations are associated with colorectal cancer with increased MYC abundance ${ }^{3,35}$. Thus, proper control of MYC transcription likely requires physical interaction between FIR and XPB ${ }^{16,23,24,43}$.

However, it is unclear whether post-initiation control via RNA Pol II pausing in the MYC promoter is required for in vivo control of $M Y C$ transcription. Intriguingly, ex vivo studies suggest physical interaction between FIR and XPB lacking the wild-type $\mathrm{C}$ terminus are defective in cells from one patient with severe $\mathrm{XP} / \mathrm{CS}$ and multiple cancers (XP11BE) ${ }^{16,24,38,40}$, but whether other $X P B$ patient mutations affect the interaction with FIR is unknown. Given the role in controlling abundance of the MYC oncogene, we hypothesized that altered interactions between XPB and FIR may contribute, at least in part, to malignancy in patients with $X P B$ mutations.

Haywire (Hay) is the Drosophila XPB homologue $40,41,43,44$, and loss-of-function Hay mutants display increased cell death, consistent with DNA repair and/or transcription defects ${ }^{41,42,46}$. The Drosophila orthologue of FIR, Half Pint (Hfp) behaves as a tumour suppressor, as loss of Hfp results in larval overgrowth and overproliferation $^{43,44}$. Hay forms a complex with Hfp in vivo and co-ablation of Hay in Hfp loss-of-function cells reduces $d M Y C$ expression and cell growth ${ }^{44,47-49}$. Altogether these data suggest conservation between mammalian FIR-XPB and Drosophila Hfp-Hay in terms of transcriptional regulation of $M Y C$ and growth control.

Here we demonstrate that C-terminally truncated Hay mutants enhance the larval overgrowth associated with reduced $\mathrm{Hfp}$ abundance $18,43,50,51$. Moreover, we show that animal overgrowth depends on elevated dMYC, thus providing further support for the homology between Hfp and FIR in regards to their capacity to repress MYC transcription via XPB/Hay ${ }^{44,52,53}$. Altogether, these data suggest mutations disrupting FIR function have the potential to promote XPB-related cancers.

\section{Results}

Hay mutants modify Hfp-dependent overgrowth. The alignment between the Human XPB-mutation associated with $\mathrm{XP} / \mathrm{CS}$ disease and the Hay mutations used here are shown in Fig. 1a. The conserved domains between the Human-XPB and Drosophila-Hay protein are highlighted and include the $\mathrm{N}$-terminal DNA binding domain, several helicase domains and the C-terminal domain. The Drosophila Hay ${ }^{n c 2}$ allele was first identified in $1988^{45,54}$ and characterized as a point mutation at amino-acid position 652 in the sixth helicase domain ${ }^{18,40,50,51,54}$. Subsequently, following ethyl methane sulfonate-induced mutagenesis, several other Hay mutants were generated in the Hay ${ }^{\text {nc } 2}$ background and molecularly characterized ${ }^{41,54}$ The original $\mathrm{Hay}^{n c 2}$ point mutation affects the conserved helicase domain but encodes an in-frame C-terminal domain, whereas the ethyl methane sulfonate mutants chosen for analysis here (Hay ${ }^{n c 2 r v 1}, H_{a y}{ }^{n c 2 r v 3}, H a y^{n c 2 r v 7}$ ) contain stop codons at position 657, 380 and 441, respectively, and lack the C-terminal domain (Fig. 1a). These mutants 
a

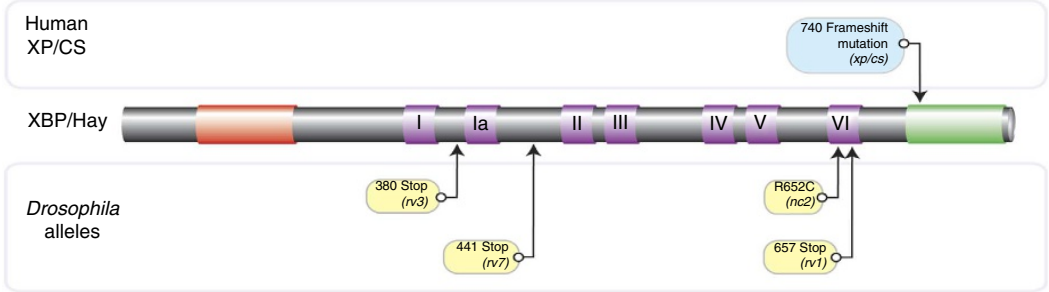

b Comparison third instar larval size

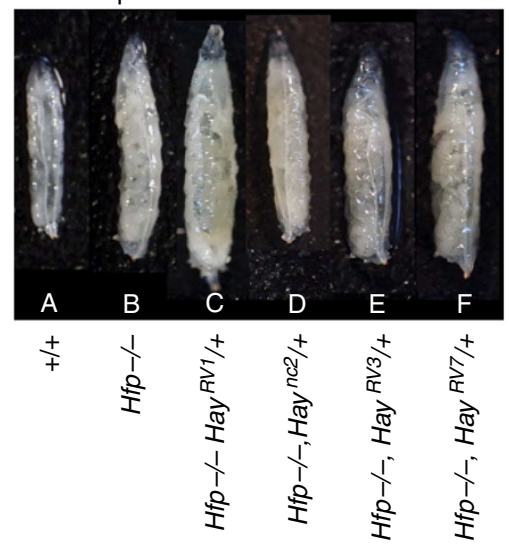

C

Quantification of larval size

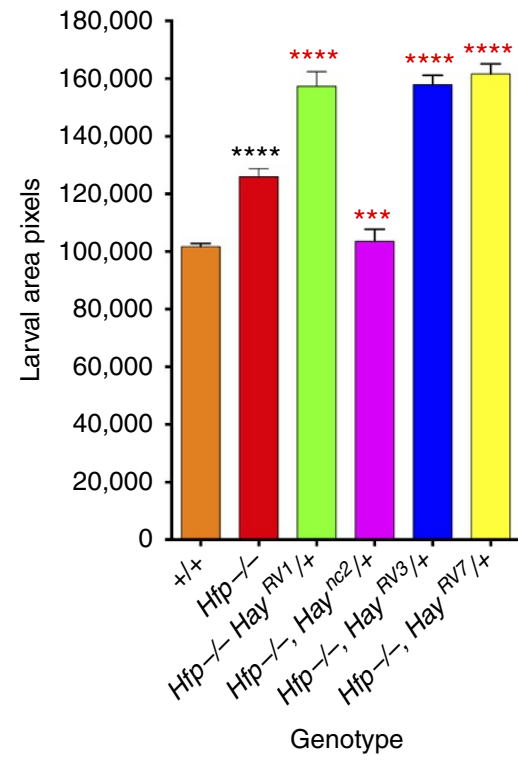

d Wing disc mitosis

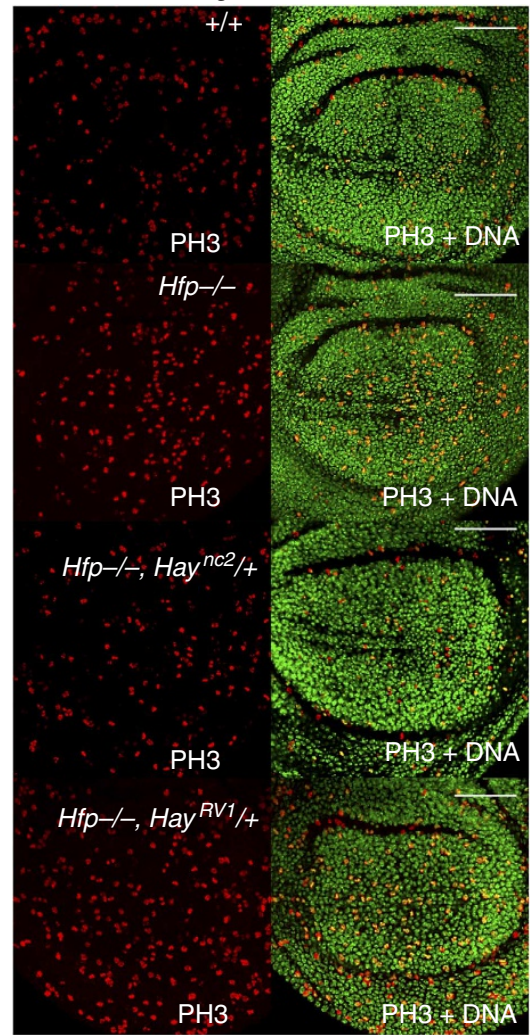

e Quantification wing disc mitosis

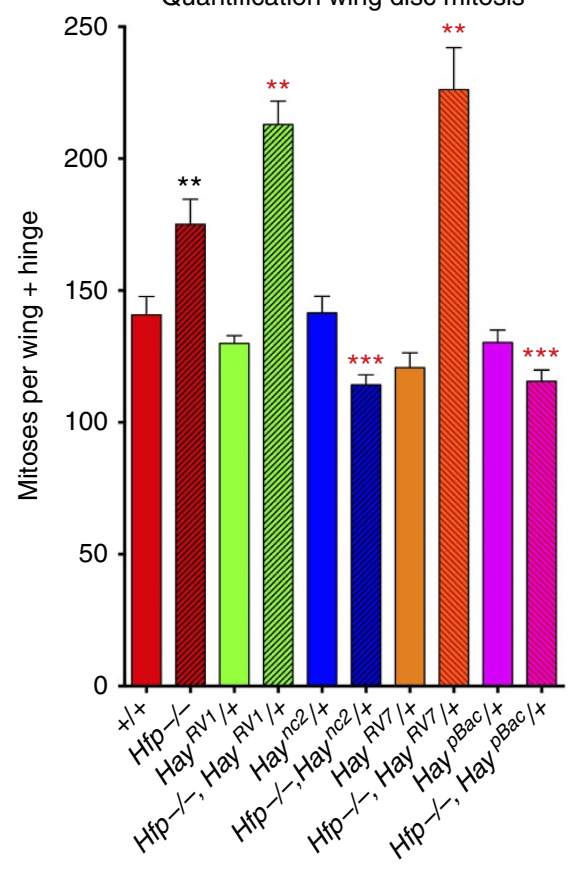

Genotype

Figure 1 | Hay mutants differentially modify overgrowth and proliferation of hypomorphic Hfp mutants. (a) Alignment of XPB and Hay showing mutations associated with XP/CS compared with Drosophila mutant alleles. Conserved domains include; N terminal (red), helicase (purple) and Carboxyterminal (green). (b) Hay mutant alleles differentially modify the third instar larval overgrowth phenotype for the Hfp hypomorph. (c) quantified larval area (see Supplementary Table 1 for quantification and number of biological replicates). (d) Hay mutant alleles differentially modify overproliferation of $H f p$ hypomorphs. Third instar larval wing imaginal discs stained with anti-phosphohistone H3 (PH3). All scale bars represent $50 \mu \mathrm{m}$. (e) quantification of mitosis (see Supplementary Table 2 for quantification and biological replicates). The black asterisks show significance points compared with control; red asterisks compared with Hfp hypomorph alone. Statistical tests for this figure and all following figures were performed with Graphpad Prism 6 using unpaired two-tailed $t$-test with $95 \%$ confidence interval. In all figures error bars represent s.e.m. and according to the Graphpad classification of significance points ${ }^{\star} P=0.01$ to $0.05,{ }^{\star \star} P=0.001-0.01,{ }^{\star \star \star} * P=0.0001-0.001$ and ${ }^{\star \star \star \star *} P<0.0001$. 
have been selected because mutations of $X P B$ encoding a $\mathrm{C}$-terminally truncated protein have been implicated in XP/ $\mathrm{CS}^{3,55}$ (Fig. 1a).

The $H f p^{\mathrm{EP}}$ contains an enhancer $\mathrm{P}$ element insertion in the $5^{\prime}$-UTR of $H f p, 94 \mathrm{bp}$ upstream of the initiating methionine and expresses reduced $H f p$ (Fig. 2) ${ }^{43,56}$. Homozygous $H f p^{\mathrm{EP}}$ larvae and pupae are $\sim 20 \%$ larger than wild-type third instar larvae, due to increased proliferation during the third larval instar (Fig. 1b-e). Homozygosity for the Hay mutant alleles is embryonic lethal, consistent with the helicase function being essential for transcription and DNA repair ${ }^{40,55}$. Although heterozygosity for the Hay alleles alone does not result in overproliferation (Fig. 1d,e and discussed below), we observed that one copy of particular Hay alleles modified the larval overgrowth phenotype associated with the hypomorphic $H f p$ mutant (Fig. 1b,c, $P<0.0001$, Supplementary Table 1) $18,43,57,58$. The $\mathrm{Hay}^{n c 2}$ allele significantly suppressed (Fig. 1b,c, $P=0.0004$ ) and, conversely, Hay ${ }^{n c 2 r v 1}$ significantly enhanced (Fig. 1b,c, $P<0.0001)$, the $H f p$ larval overgrowth phenotype. Similarly, two additional Hay alleles (Hay ${ }^{n c 2 r v 3}$ and $H a y^{n c 2 r v 7}$ ) lacking the C-terminal domain, and 5-6 of the helicase domains (that is, this deletion also removes the original point mutation at amino-acid position 652 in the sixth helicase domain), significantly enhanced the $H f p$ overgrowth phenotype (Fig. 1b,c, $P<0.0001$, Supplementary Table 1).

Suppression of growth by the Hay ${ }^{n c 2}$ point mutant is consistent with our observation that reduced Hay abundance suppressed overgrowth from loss of $\mathrm{Hfp}^{18,44,57}$. However the Hay alleles lacking the C-terminal domain behave counter to what would be predicted for loss-of-function Hay mutations, which a
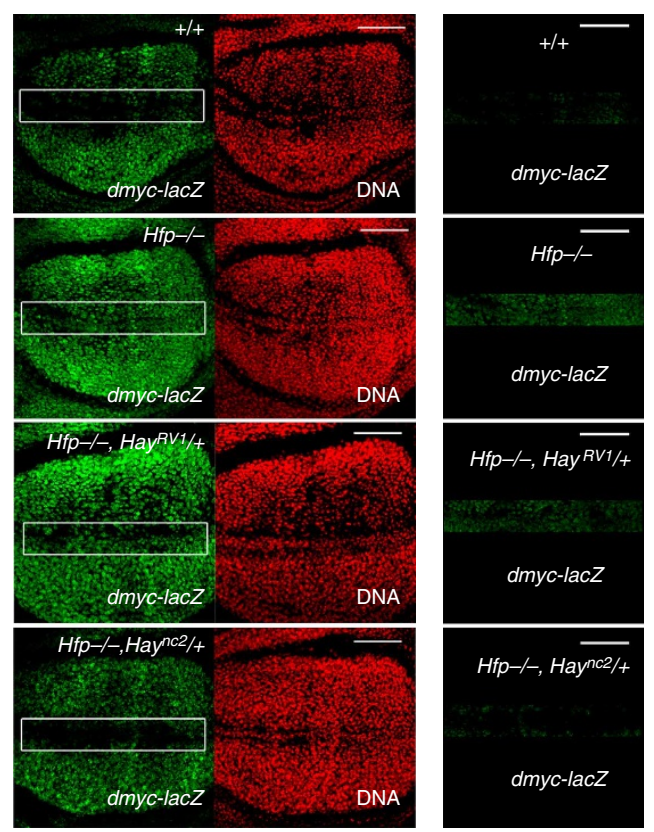

c
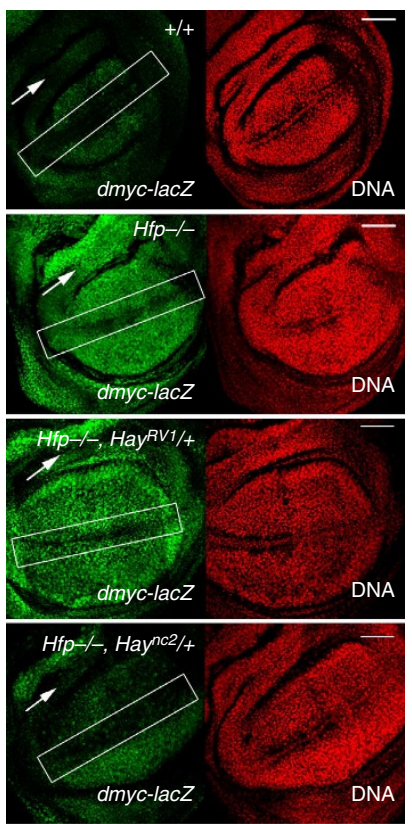

f

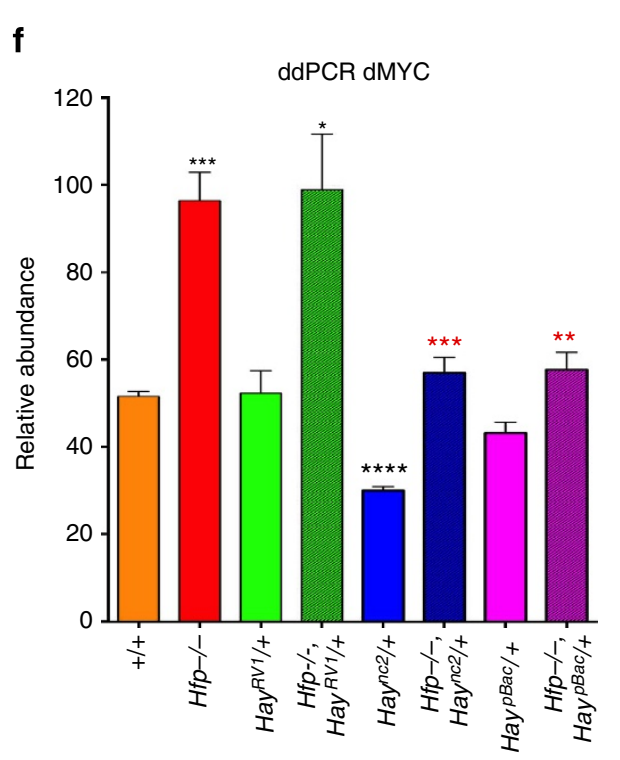

g

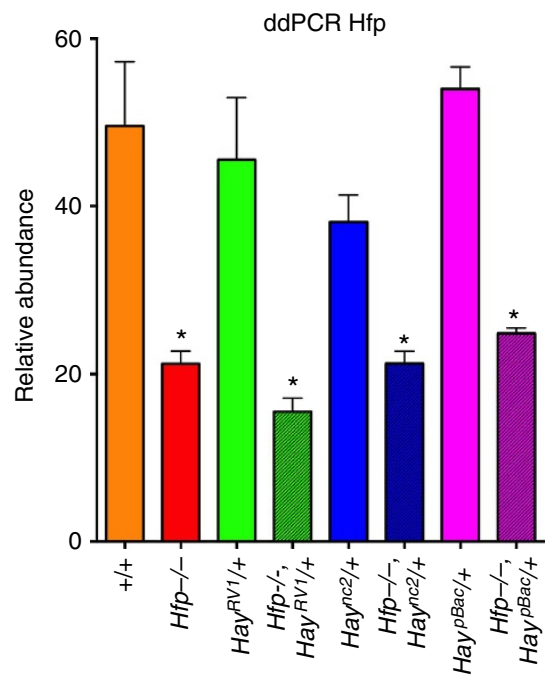

d

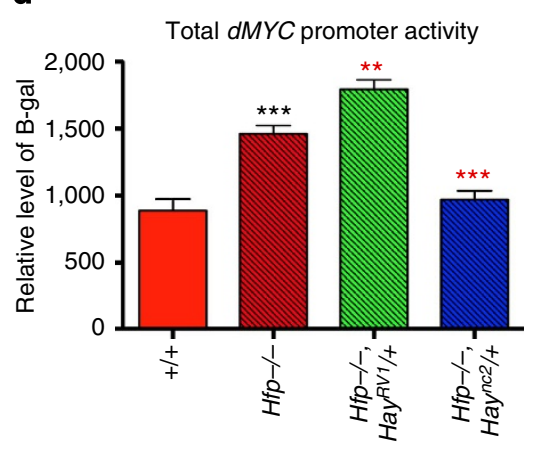

Genotype e

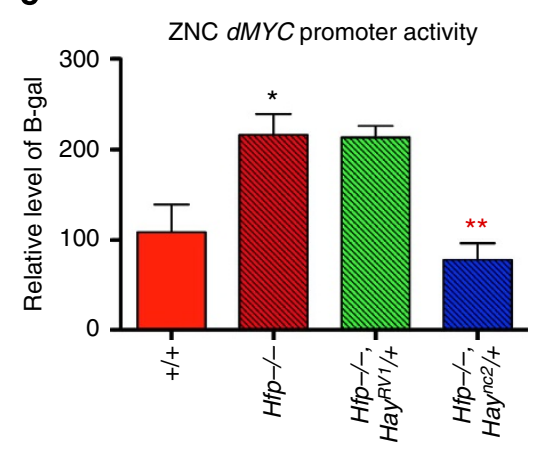

Genotype
Figure 2 | Hay mutants differentially modify dMYC-promoter activity associated with the Hfp hypomorph. (a) dMYC-lacZ activity detected with anti $\beta$-gal (green) and DAPI (red) for genotypes marked. The rectangles mark the D-V boundary. (b) $d M Y C$-lacZ across the D/V boundary. (c) lower magnification view to show $d M Y C-l a c Z$ in the hinge (marked with a white arrow in the dorsal region). All scale bars represent $50 \mu \mathrm{m}$. (d) Quantification of dMYC-lacZ activity across the wing disc and (e) across the D/V boundary. The graphs in $D$ and $E$ show the relative intensity for $\beta$-gal antibody staining (mean $\beta$-gal pixel intensity \pm s.e.m.) for each genotype (Supplementary Table 3 for quantification and number of biological replicates). (f-g) ddPCR for dMYC and Hfp mRNA abundance in wing discs, normalized to tubulin (see Supplementary Table 4 for quantification and number of biological replicates). The black asterisks show significance points compared with control; red asterisks compared with Hfp hypomorph alone. Statistical tests were performed with Graphpad Prism 6 using unpaired two-tailed t-test with 95\% confidence interval. In all figures error bars represent s.e.m. and according to the Graphpad classification of significance points ${ }^{\star} P=0.01-0.05,{ }^{\star \star} P=0.001-0.01,{ }^{\star \star \star} P=0.0001-0.001$ and ${ }^{\star \star \star \star} P<0.0001$. 
suggests the C-terminal domain may be required for growth repression. To characterize the counter-intuitive observation that the C-terminally truncated Hay mutants (Hay ${ }^{n c 2 r v 1}$, Hay ${ }^{n c 2 r v 3}$ and $H a y^{n c 2 r v 7}$ ) enhance the $H f p$ phenotype we next analysed cell cycle markers in the larval wing imaginal discs from these animals.

Hay mutants modify cell division in the $H f p$ hypomorph. To determine whether the larval overgrowth (Fig. 1b,c) was associated with increased proliferation we measured mitosis in larval wing imaginal discs. Hypomorphic $H f p$ discs had significantly more PH3 cells, compared with wild type (Fig. 1d,e, $P=0.0084$, Supplementary Table 2) and, in line with the observation that heterozygous Hay mutants do not overgrow, levels of mitosis were not significantly altered by the point mutant or C-terminally truncated Hay alleles alone (Fig. 1e). The Hay allele with the smallest C-terminal truncation (Hay ${ }^{n c 2 R V 1}$ ) significantly increased mitosis compared with the Hfp hypomorph alone (Fig. 1d,e, $P=0.0073$ ). Thus, Hay ${ }^{n c 2 R V I}$ can only promote overproliferation in the context of reduced Hfp. Similarly, the $H_{a y}{ }^{\text {nc2RV7 }}$ mutant, which lacks the C-terminal domain including the point mutation at position 652, did not result in overproliferation alone, but significantly increased mitosis in the $H f p$ hypomorph background (Fig. 1e, $P=0.0082$ ).

In contrast, the point mutant (Hay ${ }^{n c 2}$ ) significantly decreased PH3 compared with the Hfp hypomorph alone, to return mitosis to the wild type range (Fig. 1d,e, Supplementary Table 2, $P=0.0002)$. Similarly, heterozygosity for Hay loss-of-function mutations generated by P-element insertion in the Hay open-reading frame (PBac\{WH\}hayfo0028), which will lead to reduced abundance of wild type protein ${ }^{46,59}$, significantly decreased mitosis compared with $H f p$ alone (Fig. 1e, $P=0.001$ ). Altogether these data suggest repression of cell cycle by $\mathrm{Hfp}^{44,60,61}$, is sensitive to the level and function of Hay. However, since increased proliferation was not observed for the $\mathrm{C}$-terminal truncated mutants alone, this suggests the $\mathrm{C}$-terminal domain of Hay is only required for repression of cell cycle when Hfp is reduced. Thus we next investigated whether increased growth and proliferation was associated with changes to $d M Y C$ transcription.

dMYC transcription in Hfp mutants is sensitive to Hay. The wing imaginal disc is an epithelial sheet, which can be subdivided into notum, hinge and wing pouch regions, based on the fate of each of these structures in the adult. Developmental signalling events set up compartment boundaries within the wing disc to define anterior-posterior $(\mathrm{A} / \mathrm{P})$ and dorsoventral $(\mathrm{D} / \mathrm{V})$ compartments ${ }^{47-49,62}$. In particular, $d M Y C$ expression is downregulated across the $\mathrm{D} / \mathrm{V}$ boundary to form the presumptive wing margin during the third larval instar and, as a consequence, cells are delayed in the cell cycle and form a 'zone of non-proliferating cells' (ZNC) at the D/V boundary ${ }^{18,50,51,62}$ (Fig. 2a).

To investigate changes to $d M Y C$ transcription we used a $d M Y C-l a c Z$ enhancer trap, which reflects $d M Y C$ promoter activity. B-gal was detected in the cycling cells of the third instar wing pouch and reduced both along the differentiating cells at the $\mathrm{D} / \mathrm{V}$ boundary and throughout the hinge domain ${ }^{52,53,57}$ (Fig. 2a,c), consistent with patterning of $d M Y C$ mRNA $^{54,62}$. $d M Y C$ enhancer trap activity spanning the entire wing disc (pouch and hinge) was significantly greater in $H f p$ mutants compared with wild type (Fig. 2a,c, quantified in 2D, $P=0.0003$, Supplementary Table 3) and further significant increases were observed in the Hay ${ }^{n c 2 r v 1}$ background (Fig. 2a,c quantified in 2D, $P=0.0052)$. Conversely, the Hay point mutant reduced $d M Y C$ promoter activity in the $H f p$ hypomorph (Fig. 2a,c, quantified in
2D $P=0.0003)$. Altogether, these data suggest modulation of growth and proliferation in the Hfp hypomorph by the Hay alleles is due, at least in part, to differential effects on $d M Y C$ promoter activity.

As highlighted above, $d M Y C$ is downregulated along the $\mathrm{D} / \mathrm{V}$ boundary of the wing disc in preparation for cell cycle exit and differentiation of sensory bristles along the adult wing margin (rectangles in Fig. 2a,c) $)^{16,18,23,24,50,51,54}$.dMYC mRNA is detected in the second instar hinge, which forms circular domains that surround the pouch, but is normally downregulated in third instar ${ }^{44,54}$, as reflected by the pattern of $d M Y C$ enhancer trap activity (Fig. 2c, white arrow). Ectopic $d M Y C$-lacZ activity was observed along the $\mathrm{D} / \mathrm{V}$ boundary and within the presumptive hinge of $H f p$ hypomorphs (Fig. 2a-c). Significantly higher levels of promoter activity were detected throughout the $\mathrm{D} / \mathrm{V}$ boundary of $H f p$ mutants compared with the control (Fig. 2b,e, $P=0.0319$ ). Although $d M Y C$-lacZ was still detected throughout the ZNC for $H f p$ mutants in the presence of the C-terminally deleted Hay ${ }^{n c 2 r v I}$ allele, a further increase in $d M Y C$ promoter activity was not observed across the wing margin (Fig. 2b,e). The Hay point mutant significantly reduced $d M Y C$-lacZ activity compared with the Hfp mutant alone (Fig. 2b,e, $P=0.0035$, Supplementary Table 3 ), such that $d M Y C$ promoter activity was not significantly different from wild type.

We also observed continued $d M Y C$ promoter activity in the hinge of the Hfp mutant wing discs (Fig. 2c, white arrow). Suppression of $d M Y C$-lacZ activity in the hinge was observed for the Hay point mutant compared with Hfp mutant alone, however, enhancement by the C-terminally truncated mutant was difficult to ascertain as the $\beta$-gal signal was already saturated in the $H f p$ mutant background (Fig. 2c, arrows). Broadly, however, Hfp mutants alone significantly increased $d M Y C$ promoter activity, which was further increased by the C-terminally truncated Hay protein and, conversely, suppressed by the Hay point mutant.

To determine whether altered $d M Y C$ promoter activity was associated with changes to productive transcription we quantified $d M Y C$ mRNA abundance in third instar wing imaginal discs using digital PCR (with probes spanning the $3^{\prime}$ exon-intron boundary of the most $3^{\prime}$ exon). As expected $H f p$ mRNA was significantly decreased in the hypomorphic background (Fig. $2 \mathrm{~g}$, $P>0.05$ for the $H f p$ hypomorph alone and in the Hay ${ }^{n c R R V}$, $\mathrm{Hay}^{\text {nc2 }}$ and Hay ${ }^{p B a c}$ backgrounds compared with wild type, see Supplementary Table 4). Importantly, we found a significant increase in $d M Y C$ mRNA in the Hfp hypomorph compared with the wild-type control (Fig. 2f, $P=0.0005$ ). $d M Y C$ mRNA was also significantly increased in hypomorphic $H f p$ wings heterozygous for $\mathrm{Hay}^{n c 2 R V 1}$ (Fig. 2f, $P=0.0101$ compared with wild type, see Supplementary Table 4 ). The increased proliferation observed in the Hayc2rvl background was not, however, associated with a further increase in $d M Y C$ mRNA abundance. This could be a consequence of negative feedback loops, whereby increased dMYC abundance leads to autorepression at the level of transcription ${ }^{55,63}$. Specifically, and consistent with the autorepression first suggested for mammalian $M Y C^{56,64}$, overexpression of $d M Y C$ reduces endogenous $d M Y C$ transcription ${ }^{55}$. Thus, although there is a similar twofold increase in $d M Y C$ at this stage of wing development, an earlier increase in dMYC activity associated with Haync2rv1 may have induced a program of enhanced proliferation. Importantly, heterozygosity for the Hay point mutant or P-element mutant significantly decreased $d M Y C$ mRNA compared with the Hfp hypomorph alone (Fig. 2f Hay ${ }^{n c 2}$ $P=0.006$ and Hay ${ }^{p B a c}, P=0.0059$, Supplementary Table 4). Indeed $d M Y C$ mRNA levels in $H f p$ hypomorphs heterozygous for $\mathrm{Hay}^{n c 2}$ or $\mathrm{Hay}{ }^{\mathrm{Bac}}$ were not significantly different from wild type.

dMYC antibody staining of wing discs revealed that disrupted patterning of the $d M Y C$ promoter was associated with altered 
dMYC protein localization. As predicted hypomorphic $H f p$ wings from 3rd instar larvae had an overall increase in dMYC antibody staining, particularly in the ZNC and hinge (Fig. 3a). Heterozygosity for the C-terminal Hay mutant in the Hfp hypomorph further increased dMYC abundance, particularly the ectopic $\mathrm{dMYC}$ in the hinge and across the D/V boundary. As anticipated, given the decrease in proliferation in wing discs for $H f p$ mutants heterozygous for the Hay point mutant, dMYC protein was
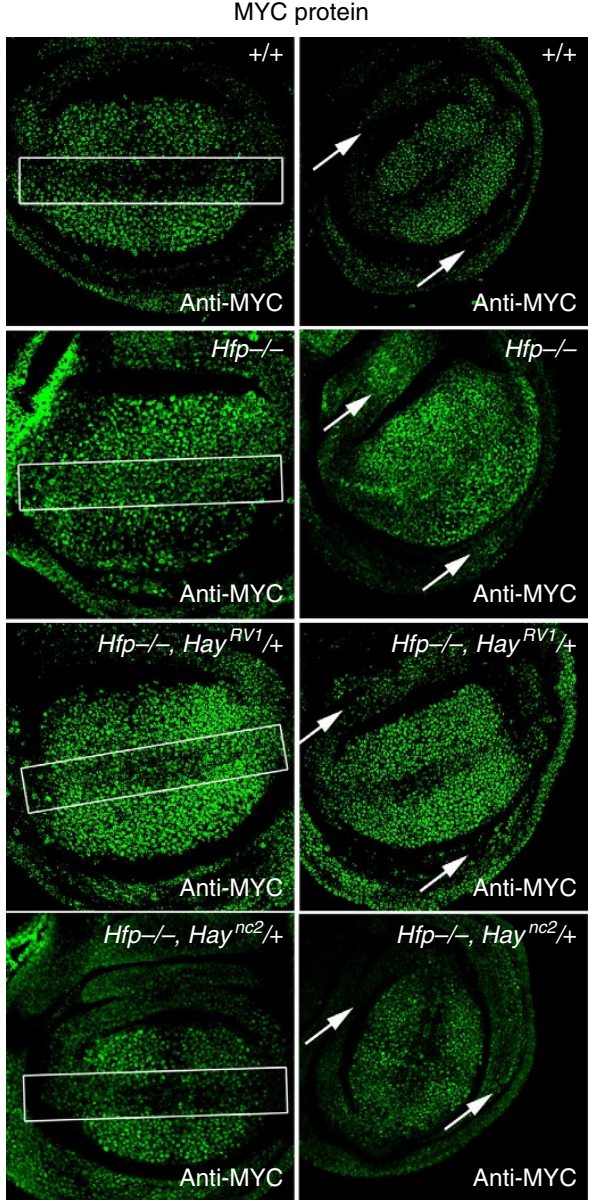

C
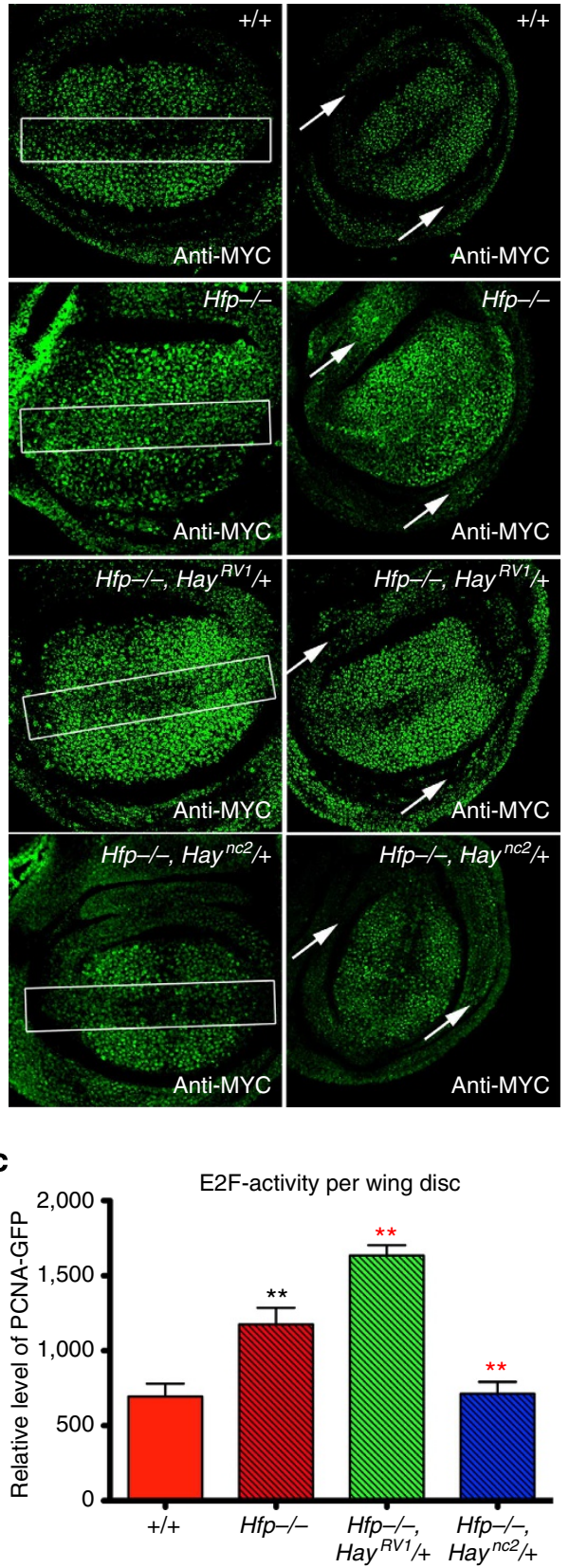

decreased, and this was particularly clear in the hinge (Fig. 3a). To determine whether the altered $d M Y C$ promoter activity, mRNA and protein abundance was associated with changes to dMYC function we next monitored $\mathrm{S}$ phase progression in wing discs.

$S$ phase activity in $H f p$ mutants is sensitive to Hay. dMYC drives cell growth and couples this with progression through the

b
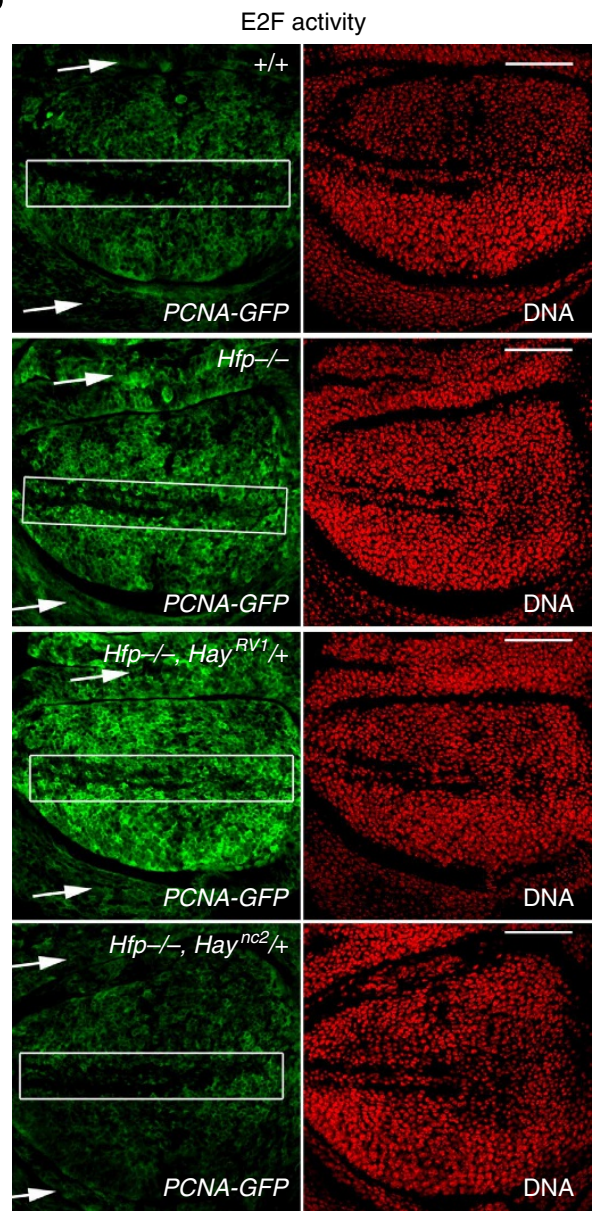

d

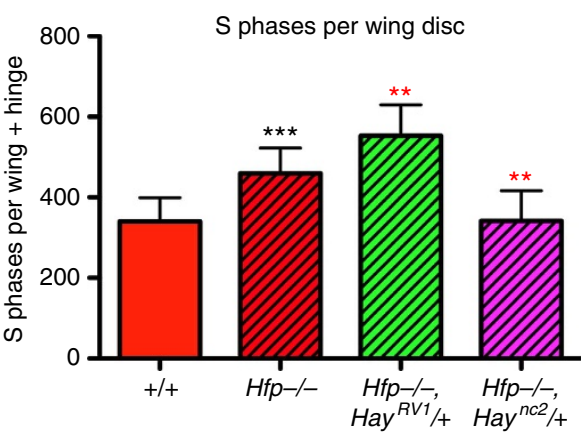

Figure 3 | Hay mutants differentially modify MYC protein patterning, E2F-activity and S phase progression in Hfp hypomorphs. (a) MYC antibody staining (green) for genotypes marked. The white rectangles mark the ZNC and white arrows mark the hinge domain. All scale bars represent 50 um. (b) PCNA-GFP activity (green) and DAPI (red) for genotypes marked. (c) Quantification of PCNA-GFP activity. The graph shows the relative intensity for GFP (mean pixel intensity \pm s.e.m.) for each genotype (see Supplementary Table 5 for quantification and number of biological replicates). (d) Quantification of $\mathrm{S}$ phase using BrdU. The graph shows number of BrdU positive cells ( \pm s.e.m.) in wing discs for genotypes marked (see Supplementary Table 6 for quantification and number of biological replicates). The black asterisks show significance points compared with control; red asterisks compared with $H f p$ hypomorph alone. Statistical tests were performed with Graphpad Prism 6 using unpaired two-tailed $t$-test with $95 \%$ confidence interval. In all figures error bars represent s.e.m. and according to the Graphpad classification of significance points ${ }^{\star} P=0.01-0.05,{ }^{\star \star} P=0.001-0.01,{ }^{\star \star \star} P=0.0001-0.001$ and $\star \star \star \star * P<0.0001$ 
cell cycle via activation of $S$ phase genes ${ }^{18,57,58}$. In particular, dMYC promotes initiation of DNA synthesis/S phase via upregulation of the G1-S cyclins, Cyclin E and Cyclin $\mathrm{D}^{18,57}$, which pair with the Cyclin-dependent kinases (CDKs), Cdk2 (ref. 59) and Cdk4 (refs 60,61), respectively. The Cyclin/CDK complexes can phosphorylate and inactivate the Retinoblastoma protein $(\mathrm{Rb})$ homologue $\mathrm{Rbf}$ to release the $\mathrm{E} 2 \mathrm{~F}$ transcription factor to activate expression of the $S$ phase genes ${ }^{62}$. A key target of $\mathrm{E} 2 \mathrm{~F}$ and its binding partner DP is the DNA replication factor, proliferating cell nuclear antigen (PCNA $)^{62}$. Increased dMYC activity will, therefore, lead to upregulation of E2F1 function ${ }^{57}$, which can be measured using a PCNA-GFP reporter ${ }^{62}$.

The PCNA-GFP pattern in wing discs shows E2F activity throughout dividing cells in the wing pouch and the expected decrease across the D/V boundary in wild-type discs (Fig. 3b). In line with the abnormal pattering of the $d M Y C$ enhancer trap, increased $A M Y C$ mRNA (Fig. 2) and protein (Fig. 3a), ectopic PCNA-GFP activity was observed across the presumptive wing margin and throughout the hinge of Hfp mutant discs (Fig. 3b, Supplementary Figure 1) and GFP intensity was significantly greater in the $H f p$ hypomorph compared with wild type (Fig. 3c, $P=0.009$, see Supplementary Table 5). In accordance with the C-terminally truncated Hay allele further increasing dMYC activity, PCNA-GFP activity was significantly increased across the wing pouch, compared with the $H f p$ mutant alone $(P=0.0028$, Fig. 3c). Conversely, the Hay point mutant significantly decreased E2F-activity in the $H f p$ mutant background $(P=0.0070$, Fig. $3 \mathrm{c})$. Moreover, the Hay ${ }^{n c 2}$ point mutant reduced PCNA-GFP activity across the ZNC (Fig. $3 \mathrm{~b}$ ) and the hinge (Supplementary Figure 1). The increased PCNA-GFP activity was also associated with cell cycle progression; BrdU incorporation revealed that $H f p$ mutants had significantly more cells in S phase than control (Fig. 3d, $P=0.0004$, see Supplementary Table 6). $S$ phases were further increased by the $H a y^{n c 2 R V 1} \mathrm{C}$-terminal deletion and reduced by the Hay ${ }^{n c 2}$ point mutant (Fig. $3 \mathrm{~d}, P=0.0077$ and $P=0.0022$, respectively). Thus the C-terminally truncated Hay protein further increases $d M Y C$ promoter activity and $\mathrm{S}$ phase progression, suggesting increased dMYC-activity drives the overproliferation and larval overgrowth and wing disc overproliferation in the $H f p$ mutant background (Fig. 1).

dMYC is critical for Hfp and Hay dependent overgrowth. We next demonstrated that the overgrowth associated with $H a y^{n c 2 R V 1}$ was dependent on reduced Hfp and/or elevated dMYC. As shown above, $H f p$ mutants alone, or in the heterozygous $H a y^{n c 2 R V 1}$ background, were significantly larger than wild type (Fig. 1b,c, $P<0.0001$ for both). Ubiquitous overexpression of $H f p$ from the enhancer $\mathrm{P}$, which is inserted in the Hfp promoter and contains UAS sites, with the Arm-GAL4 driver (Supplementary Table 7 and Supplementary Figure 2 for quantification of $H f p$ and $M Y C$ mRNA) resulted in significantly reduced overgrowth of hypomorphic $H f p$ larvae, both alone and in the $H a y^{n c 2 R V 1}$ background (Fig. $4 \mathrm{a}, \mathrm{c}, P=0.0331$ and $P=0.0318$ compared with $H f p$ alone, Supplementary Table 8). Thus, overgrowth in these animals was dependent on decreased abundance of Hfp.

Importantly, overgrowth of $H f p$ hypomorphs, either alone or in the $H a y^{n c 2 R V 1}$ background, was dependent on elevated dMYC. Specifically, heterozygosity for the $d M Y C$ mutant $\left(d m^{P 0}\right)$, which returned $d M Y C$ mRNA levels to the wild-type range in wing imaginal discs (Fig. 4b; Supplementary Table 9), significantly decreased larval growth (Fig. 4a,c $P=0.025$ for $H f p-/-$ and $P<0.0001$ Hfp $-/-$, Hay ${ }^{n c 2 R V 1}$, Supplementary Table 8). Mitosis was also returned to the wild-type range in wing imaginal discs heterozygous for an alternate $d M Y C$ mutant $\left(\mathrm{dm}^{\mathrm{G0354}}\right.$;
Fig. $4 \mathrm{c}, P=0.0132$ for $H f p-I-$ and $P<0.0001$ for $H f p-I-$, $H_{a y}{ }^{n c 2 R V 1}$ Supplementary Table 10). Altogether these data suggest that the increased animal overgrowth and overproliferation in the Hfp hypomorph, and further growth driven by $H a y^{n c 2 R V 1}$, is dependent on elevated dMYC.

Hay mutants differentially alter RNA Pol II pausing on $d M Y C$. Control of mammalian $M Y C$ transcription requires interaction between the Hfp (FIR) and Hay (XPB) orthologues ex vivo 16,23,24, thus, we hypothesized that the Hay-Hfp interaction ${ }^{44}$ might regulate transcription in vivo via RNA Pol II activity within the $d M Y C$ promoter. We therefore used ChIP to monitor RNA Pol II enrichment across the $d M Y C$ promoter, using antibodies specific to the C-terminal domain (CTD) of the largest RNA Pol II subunit (Fig. 5). RNA Pol II is recruited to gene promoters in a hypophosphorylated (inactive) state and promoter escape requires TFIIH-mediated phosphorylation of the Ser5 residue of the CTD of the largest subunit ${ }^{63}$. Thus antibodies specific to (1) the Ser-5 phosphorylated isoform and (2) all isoforms of the CTD were used for ChIP.

ChIP from wild-type imaginal discs revealed a peak of RNA Pol II (Ser5 and total) proximal to the $d M Y C$ transcriptional start site (Fig. 5b,c, blue columns), which suggests $d M Y C$ transcription is regulated by RNA Pol II pausing in vivo. The RNA Pol II detected using the total RNA Pol II antibody is therefore likely to be predominantly Ser5 phosphorylated, suggesting most of the RNA Pol II on $d M Y C$ is normally poised for promoter escape and transcription. In contrast to control, the Hfp hypomorph had significantly decreased Ser5 and total RNA Pol II enrichment across the $d M Y C$ TSS (Fig. 5b, $P=0.0052$, Fig. 5c, $P=0.0007$, respectively Supplementary Tables 11,12$)$. This suggested reduced levels of Hfp, which are associated with increased $d M Y C$ expression, impair RNA Pol II pausing across the $d M Y C$ promoter. Thus we investigated whether the Hay mutants might differentially affect RNA Pol II to account for the altered $d M Y C$ promoter activity/transcription (Fig. 2).

Interestingly, the Hay ${ }^{n c 2 R V 1}$ C-terminal deletion further decreased enrichment for both Ser5 and RNA Pol II (Fig. 5b, $P<0.0001$ ) and total RNA Pol II (Fig. 5c, $P=0.0018$ ) in the $H f p$ hypomorphic background (that is, compared with $H f p$ alone), suggesting a further reduction in RNA Pol II pausing might explain the increased dMYC activity (Fig. 2). However, the observation that there is less Ser 5 RNA Pol II and total RNA Pol II on the $d M Y C$ promoter for the Hay ${ }^{n c 2 R V 1}$ C-terminal mutant alone, but no increase in $d M Y C$ expression or cell growth, suggests that the decrease in paused RNA Pol II alone is not sufficient to hyperactivate $d M Y C$. Rather, heterozygosity for the C-terminal truncation sensitizes $d M Y C$ to further hyperactivation when Hfp levels are reduced. Altogether this suggests that Hfp levels are key to the hyperactivation of $d M Y C$ transcription, as reduced RNA Pol II pausing alone is not sufficient for $d M Y C$ promoter activation when Hfp is abundant.

Although the Hay ${ }^{n c 2}$ point mutant was also associated with a further decrease in Ser5 RNA Pol II enrichment across the TSS in both the wild type and the Hfp mutant background (Fig. 5b, $P<0.0001$ for both $\mathrm{Hay}^{n c 2}$ and $\mathrm{H} f \mathrm{p}-\mathrm{I}-\mathrm{Hay}^{n c 2}$ compared with control), enrichment for total RNA Pol II was in the control range (Fig. 5c). Together this suggests nonphosphorylated and, therefore, inactive/paused RNA Pol II holoenzyme accumulates on the $d M Y C$ TSS in the Hay ${ }^{n c 2}$ background. Thus, although RNA Pol II loading occurs in these mutants, the fraction of Ser5 phosphorylated isoform appears to be reduced relative to holoenzyme, which would be expected to reduce RNA Pol II pause release from the $d M Y C$ promoter and impair transcription (that is, as observed in Fig. 2). 
a

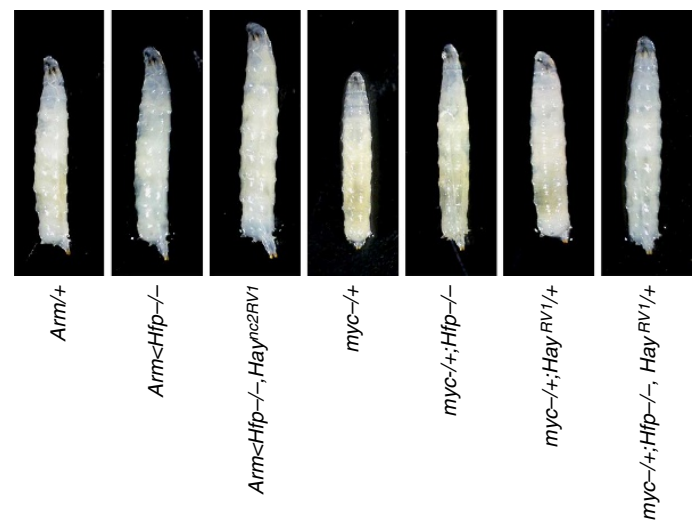

b

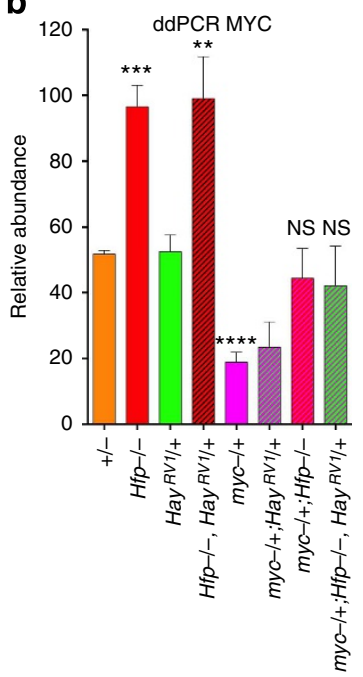

C

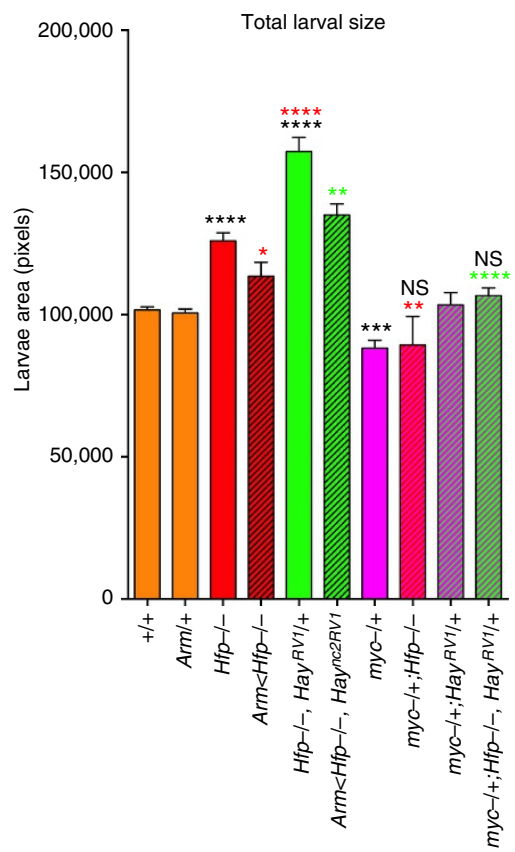

d

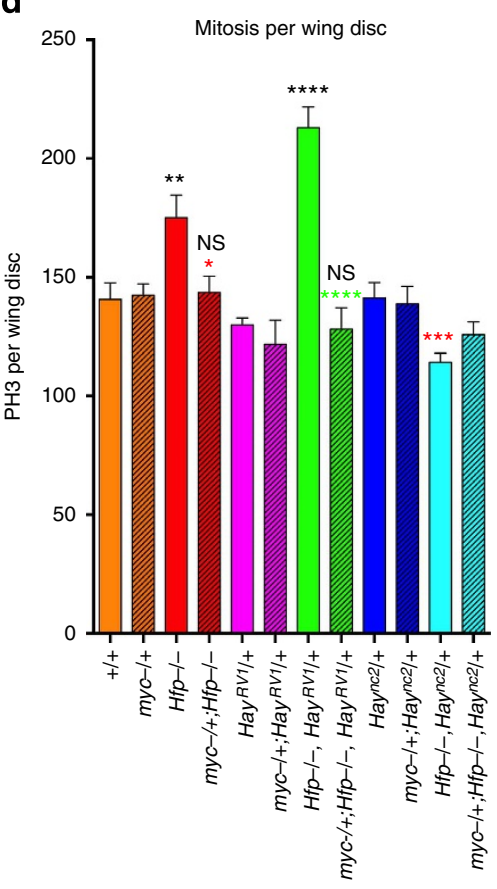

Figure 4 | Larval overgrowth and proliferation is dependent on dMYC. (a) Light microscope images of third instar larvae for genotypes marked. (b) ddPCR for $A M Y C$ and Hfp mRNA abundance in wing discs, normalized to tubulin (see Supplementary Table 9 for quantification and number of biological replicates). (c) Quantification of larval area. The graph shows the mean pixel area ( \pm s.e.m., see Supplementary Table 8 for quantification and number of biological replicates). (d) Quantification of mitosis per wing disc for the genotypes marked. The graph shows the mean PH3 count ( \pm s.e.m.; see Supplementary Table 10 for quantification and number of biological replicates). The black asterisks show significance points compared with control; red asterisks compared with Hfp hypomorph alone and green asterisks compared with Hay ${ }^{\text {nc2RV } 1}$ in the Hfp hypomorph background. Statistical tests were performed with Graphpad Prism 6 using unpaired two-tailed $t$-test with 95\% confidence interval. In all figures error bars represent s.e.m. and according to the Graphpad classification of significance points ${ }^{\star} P=0.01-0.05,{ }^{\star \star} P=0.001-0.01,{ }^{\star \star \star} P=0.0001-0.001$ and ${ }^{\star \star \star \star} P<0.0001$.

The significant reduction in $d M Y C$ expression in the Hay ${ }^{n c 2}$ mutants compared with reduced Hay abundance, which does not alter $d M Y C$ mRNA levels (that is, using the Hay ${ }^{p B a c}$ mutant, Fig. 2f), might be explained by the initial molecular characterization demonstrating the $\mathrm{Hay}^{n c 2}$ allele encodes a stable protein, that is much more abundant than the wild-type Hay protein ${ }^{41}$. Hay ${ }^{n c 2}$ might, therefore, be expected to not only impair TFIIH activity, but to also block activity of the wild type protein. Thus we predict the Hay ${ }^{n c 2}$ mutant inhibits precocious release of RNA Pol II and activation of $d M Y C$ normally associated with reduced Hfp repressor, as non-functional, stable protein blocks wild type Hay function.
Together these data provide an explanation for how the Hay alleles differentially modify dMYC-dependent overgrowth in the Hfp hypomorph; (1) being enhanced by the Hay ${ }^{n c 2 R V 1}$ C-terminal deletion due to further impairment of $d M Y C$ repression and (2) being suppressed by the $\mathrm{Hay}^{n c 2}$ point mutant due to increased accumulation of inactive RNA Pol II on the $d M Y C$ promoter.

\section{Discussion}

The XPB helicase is a multi-function protein, being required to unwind DNA before all RNA Pol II-dependent transcription ${ }^{64}$, 
a

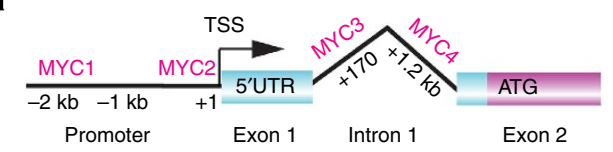

b

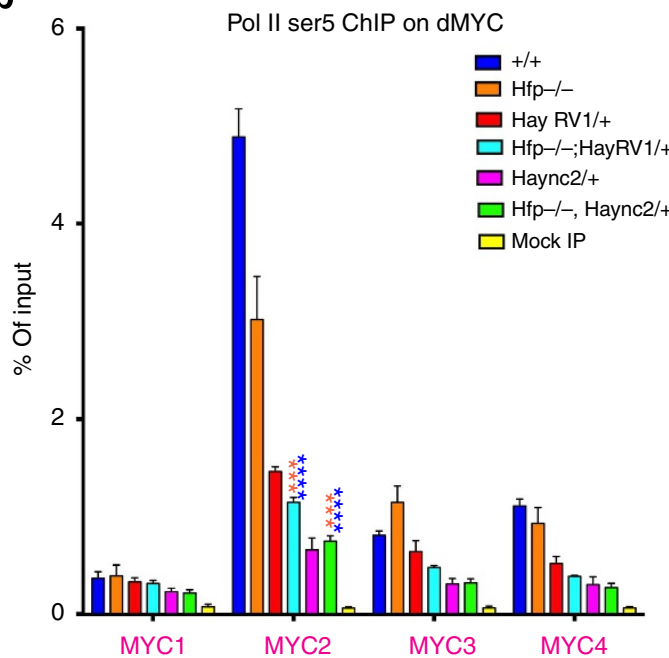

C

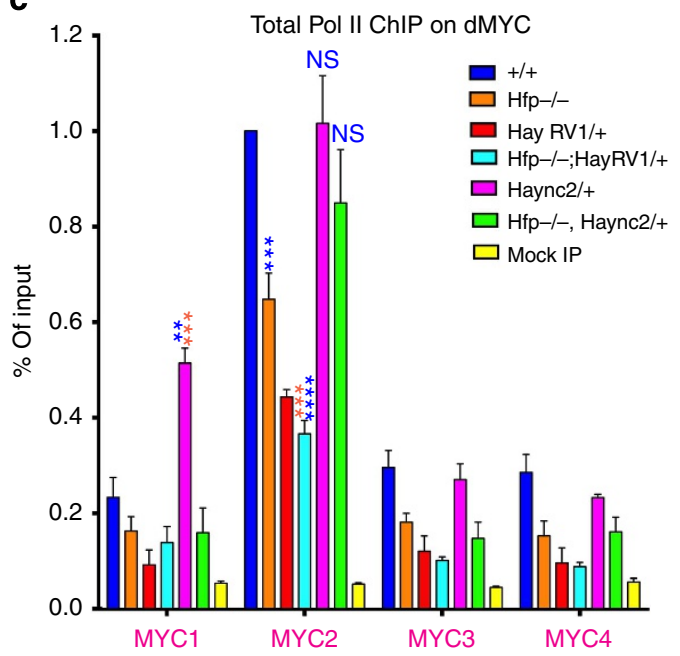

Figure 5 | Hay mutants affect RNA Pol II enrichment across the dMYC promoter. (a) Schematic of $d M Y C$ showing the position of the amplicons used for qPCR. (b) ChIP for Ser 5 RNA Pol II and (c) ChIP for total RNA Pol II (see Supplementary Table 11,12 for quantification and number of biological replicates). Statistical tests were performed with Graphpad Prism 6 using unpaired two-tailed $t$-test with $95 \%$ confidence interval. In all figures error bars represent s.e.m. and according to the Graphpad classification of significance points ${ }^{\star} P=0.01-0.05,{ }^{\star \star} P=0.001-0.01,{ }^{\star \star \star} P=0.0001-0.001$ and ${ }^{\star \star \star \star} P<0.0001$.

and for both transcription-coupled repair and global genome repair of DNA damage $2,4,6,65$. The complex disease phenotypes in $\mathrm{XP} / \mathrm{CS}$ families are emphasized by patients carrying C-terminal mutations in $X P B$, who have combined Xeroderma pigmentosum/Cockayne syndrome (XP/CS), with or without cancer ${ }^{3,9,10}$. In vivo mouse models carrying the $\mathrm{C}$-terminal $X P B$ mutation have failed to elucidate clear mechanisms underlying cancer phenotypes in XPB-related diseases. XPB/XPCS knockin mice developed to mimic XPB-related XP/CS, by encoding a C-terminally truncated protein, are indistinguishable from wildtype animals. Intriguingly, challenging the DNA repair pathways by placing the XPB/XPCS heterozygotes in an NER-deficient background (double mutant for the Xpa repair protein) did not significantly increase spontaneous tumour incidence ${ }^{11,66}$. Thus mammalian studies have left unresolved the question of why mutations in the C-terminal domain of $X P B$ result in cancer in some patients, but not others. On the basis of our Drosophila models, we predict that malignancies observed in cancer prone

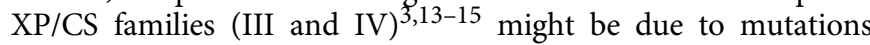
affecting FIR abundance, which we would expect to promote MYC-dependent overproliferation in the context of the XPB C-terminal mutation. In contrast we would predict that the $\mathrm{XP} / \mathrm{CS}$ family V, which has not shown cancer phenotypes $3,17,18$, has normal FIR abundance/function.

Interestingly of the other TFIIH components associated with TTD, XP, CS or XP/CS, the XPD helicase is implicated in all three diseases, including XP with increased ultraviolet-induced cancer incidence, while the TTDA protein has only been linked to the developmental disease TTD. In the repair pathway, the XP-related NER endonucleases (XPG and XPF) also only have increased cancer incidence in response to ultraviolet-irradiation. Our observations from Drosophila suggest that the increased propensity of XPB patients with the C-terminal truncation to cancer might be due to specific roles for $\mathrm{XPB}$ in mediating Hfp/FIR-dependent repression of MYC transcription.

The Drosophila models demonstrate that like the heterozygous $\mathrm{XPB} / \mathrm{XPCS}$ knockin mice, Hay ${ }^{n c 2 R V 1}$ heterozygotes do not overproliferate, but enhance cell and tissue overgrowth in a manner dependent on reduced abundance of the $d M Y C$ repressor, Hfp. The interaction between Hay and Hfp is of particular interest as, like Hay/XPB, Hfp has been implicated as a dual function protein. Initially, due to the presence of the RNA recognition motif it was implicated in RNA splicing in Drosophila ${ }^{19,67}$. However, our work suggests Hfp also behaves as a $d M Y C$ transcriptional repressor, likely via binding singlestranded DNA to maintain RNA Pol II pausing on the $d M Y C$ promoter. In mammals, FIR splice variant mutations lacking exon 2, which encodes the transcriptional repression domain, are associated with colorectal cancer and are hypothesized to promote tumour development by disrupting FIR-dependent $M Y C$ repression ${ }^{28}$. In accordance with $d M Y C$ being the core target in flies, we demonstrate overgrowth in the Hfp hypomorph, either alone or in the C-terminally truncated Hay background, is associated with defective repression of $d M Y C$ promoter activity and dependent on elevated dMYC.

The diminished enrichment of RNA Pol II across the $d M Y C$ TSS, both in the Hfp hypomorph alone, and exacerbated by the Hay C-terminal truncation, suggests the interaction between Hay and the Hfp-repressor might be required for maximal RNA Pol II pausing. This is supported by the observations that in cells from an XP/CS patient with severe and multiple cancers (XP11BE), where the XPB protein lacks the wild-type C terminus; (1) MYC transcription is dysregulated, (2) the direct interaction (via GST pull-down assays) normally observed between XPB and FIR is not detected for XPB protein and (3) FIR enrichment on MYC is maintained at the far upstream sequence element, but decreased at the promoter, suggesting impaired promoter looping $16,21,23,24$. Consistent with this, the Hay ${ }^{n c 2 R V 1}$ C-terminal mutant significantly decreases RNA Pol II enrichment on the $d M Y C$ promoter compared with the Hfp hypomorph alone (Fig. 5), and is associated with increased $A M Y C$ promoter activity, and dMYC-dependent animal growth and proliferation (Figs 1-4).

The notion that paused RNA Pol II can behave as an insulator to suppress inappropriate transcription from paused promoters $^{14,15,20,22}$ is supported by the enhancement of $d M Y C$ expression and overgrowth correlating with a further reduction in RNA Pol II enrichment on the $d M Y C$ promoter. Moreover, 
accumulation of nonphosphorylated RNA Pol II across the $d M Y C$ promoter in the Hay ${ }^{n c 2}$ background is associated with decreased $d M Y C$ transcription, both alone and in the Hfp mutant background. Hfp-dependent transcriptional mechanisms, therefore, underlie the role of Hay in controlling dMYC abundance and animal growth in vivo. In particular, the interaction between Hay and Hfp is required to maintain RNA Pol II pausing, which we predict will normally block inappropriate transcription from the $d M Y C$ promoter.

Altogether the data suggest that dysregulated transcriptional repression of MYC by the human Hfp orthologue, FIR, in addition to defective DNA repair, might also contribute to cancer progression in XPB-related human diseases. These predictions are consistent with human ex vivo experiments suggesting physical interaction between FIR, the Hfp orthologue and MYC repressor, and XPB is defective in cell lines derived from a cancer prone $\mathrm{XP} / \mathrm{CS}$ patient with the C-terminal XPB mutation ${ }^{16,24-32}$. Our observation that a single copy of the C-terminally truncated XPB homologue, Hay, increases abundance of $\mathrm{dMYC}$ and cell cycle progression in Hfp hypomorphs suggests that loss of this MYCrepressor confers susceptibility to overgrowth in vivo. Specifically, the data suggest that impaired RNA Pol II pausing and precocious RNA Pol II release in the Hfp mutant background is exacerbated by the C-terminal Hay mutant, to result in further $d M Y C$ transcription and overproliferation. Finally, our findings suggest the interaction between Drosophila Hay/Hfp and mammalian XPB/FIR is likely conserved and, therefore, that loss-of-function FIR mutations might dictate whether certain XP patients are more likely to develop neoplastic malignancies.

\section{Methods}

Fly strains. Unless otherwise stated, the Drosophila strains were obtained from the Bloomington Stock Centre. PBac $\{\mathrm{WH}\}$ hayf00028 was from the Genomic mapping of Exelixis insertion collection ${ }^{33,34,46}$. The $d M Y C$ RNAi line (v2948) was obtained from the Vienna Drosophila RNAi Center ${ }^{36,68}$. PCNA-GFP was a gift from R. Duronio.

Immunohistochemistry. Larval imaginal tissues were fixed for $30 \mathrm{~min}$ in $4 \%$ PFA, blocked in 5\% BSA in PBST at room temperature for $1 \mathrm{~h}$ before incubation overnight at $4{ }^{\circ} \mathrm{C}$ with the primary antibody and detection with fluorescently tagged secondary antibody. Primary antibodies included; $\beta$-gal (Sigma) (1:500); pH3 (millipore, 1:1,000), BrdU (Becton Dickinson, 1:100), dMYC (Santa Cruz $(1 / 200)$ and anti-GFP (to detect PCNA-GFP only, Invitrogen). After counterstaining with DAPI and placing in $80 \%$ glycerol, wing imaginal discs were dissected and imaged with the Zeiss Imager $Z$ confocal microscope using Zen Meta software. $Z$-series with $0.5 \mu \mathrm{m}$ sections were performed at $40 \times$ magnification. Fluorophores were imaged using band-pass filters to remove cross-detection between channels. Images were processed and prepared using Image J $1.43 \mu$, and Adobe Photoshop CS6 Version 11.0.2. Pixel intensity for PCNA-GFP and $d M Y C$-lacZ activity was calculated using ImageJ from $3 \times 0.5 \mu \mathrm{m}$ confocal $\mathrm{Z}$ stacks (each genotype imaged at the same pinhole and gain) from the surface of the wing imaginal disc epithelium. Mitoses were quantified by measuring the ratio of average phosphohistone $\mathrm{H} 3$-positive cells per fixed area in the PC compared to the AC over at least 7 discs. Statistical tests were performed with Graphpad Prism 6 using unpaired 2 -tailed t-test with $95 \%$ confidence interval.

Bromodeoxyuridine labelling. Larval imaginal tissues were incubated for $30 \mathrm{~min}$ in $100 \mu \mathrm{g} \mathrm{ml}^{-1} \mathrm{BrdU}$ in Schneider's media with $10 \%$ FCS followed by fixation in $4 \%$ PFA. Tissues were incubated in PBST at $37^{\circ} \mathrm{C}$ for $30 \mathrm{~min}$ followed by DNAse treatment at $37^{\circ} \mathrm{C}$ for $1.5 \mathrm{~h}$ and immunostained as described above with primary antibody for BrdU (Becton Dickinson, 1:100). Ratio of average BrdU-positive cells per fixed area in the PC compared with $\mathrm{AC}$ was calculated over at least seven discs. Statistical tests were performed with Prism 6 using unpaired two-tailed $t$-test with $95 \%$ confidence interval.

CDNA synthesis and Droplet Digital PCR. Third instar wing discs (10 pairs per genotype) were collected, RNA was extracted (Promega ReliaPrep RNA Cell Miniprep system) and eluted in $30 \mu \mathrm{l}$ nuclease-free water. RNA purity and integrity was assessed using an automated electrophoresis system (2200 TapeStation, Agilent Technologies). Six microlitres of the eluted RNA samples were used for cDNA synthesis (Bioline Tetro cDNA Synthesis kit). For each genotype ddPCR (Droplet Digital PCR) was carried out on biological triplicates and quantified using the
QX100 Droplet Digital PCR system (Bio-Rad) following the Digital MIQE guidelines ${ }^{7,69}$. The ddPCR mix consisted of: $10 \mu \mathrm{l} 2 \times \mathrm{ddPCR}^{\mathrm{TM}}$ super mix for probes (Bio-Rad); $500 \mathrm{nM}$ of primers for MYC and HFP, $250 \mathrm{nM}$ of probe mix for MYC and HFP (Integrated DNA Technologies) and $1 \mu \mathrm{l}$ of cDNA into a final volume of $20 \mu \mathrm{l}$. The total mix was placed into the eight-channel cartridge, $70 \mu \mathrm{l}$ of droplet generating oil was added and droplets were formed in the QX100droplet generator (Bio-Rad). Droplets in oil suspensions were transferred to a 96 well plate and placed into the $\mathrm{C} 1000$ Touch $^{\mathrm{TM}}$ Thermal Cycler (Bio-Rad). Cycling conditions were as follows: $95^{\circ} \mathrm{C}$ for $10 \mathrm{~min}$, followed by 40 cycles of $94^{\circ} \mathrm{C}$ for $30 \mathrm{sec}$ and $60^{\circ} \mathrm{C}$ for $60 \mathrm{~s}$. The droplets were subsequently read automatically by the QX100 droplet reader (Bio-Rad) and the data were analysed with the QuantaSoftsoftware 1.3.2.0 (Bio-Rad).

The primers used were;

MYC-5'-GTGGACGATGGTCCCAATTT-3'

$3^{\prime}$-GGGATTTGTGGG TAGCTTCTT-5';

HFP $-5^{\prime}$-CCAGCATGAATCTCTTTGATTTGG- $3^{\prime}$

$3^{\prime}$-GGAGTTCGTTGTGGGACAG-5'

The probes used were; MYC -

$5^{\prime}$-/56-FAM/CCCTCAGAT/ZEN/TCCGATGAGGAAATCGA/3IABkFQ/- $3^{\prime}$ and HFP $-5^{\prime}$-/5HEX/TCGATCTAT/ZEN/TACGCCGCCGAATGC/3IABkFQ/-3'

Chromatin Immunoprecipitation. ChIP assays were carried out as described with modifications ${ }^{37,39,70}$. For each ChIP sample, 30 larval heads were fixed in $4 \%$ paraformaldehyde for $20 \mathrm{~min}$. Larval heads were then mashed and chromatin sheared in $0.4 \%$ SDS using a Covaris S2 (10 min duration, 10\% DUTY, 200 cycles per burst, Intensity 4, achieving average DNA fragment sizes 200-600 bp). ChIPs were performed in IP buffer containing $0.1 \%$ SDS and $3 \mu \mathrm{g}$ of antibody was used for each IP (anti-RNA Polymerase II phospho S5 antibody (ab5131) or Total RNA Polymerase CTD repeat 8WG16 (ab817, Abcam). Analysis was performed in triplicate using SYBR green dye (Bioline) on the ABI Prism 7900ht (Applied Biosystems). To calculate the percentage of total DNA bound, non-immuno precipitated input samples from each condition were used as reference for all qPCR reactions. The primers for $\mathrm{qPCR}$ were;

MYC1-5'-GGCGATCGTTTCTGGCCTACGG- ${ }^{\prime}$

$3^{\prime}$-GCAGGCGCATTTGACTCGGC-5';

MYC2-5'-TTTCTACCATGCGGCCTAAC- $3^{\prime}$

$3^{\prime}$-GCTTTGGCGCCTTACATTTC-5'

MYC3 - $5^{\prime}$-ACTACTACTAACAACTGTCACAAGCCAAGT-3'

$3^{\prime}$-TTTATGTATTTGCGCGGTTTTAAG-5';

MYC4-5' ${ }^{\prime}$-TTCAAAATAGAATTTCTGGGAAAGGT- $3^{\prime}$

$3^{\prime}$ GCGGCCATGATCACTGATT $5^{\prime}$

MYC5-5'-GGTTTTCCTTTTATGCCCTTG-3'

$3^{\prime}$-CTATTAACCATTTGAACCCGAAATC-5'

\section{References}

1. Kraemer, K. H. et al. Xeroderma pigmentosum, trichothiodystrophy and Cockayne syndrome: a complex genotype-phenotype relationship. Neuroscience 145, 1388-1396 (2007).

2. Chopra, V. S., Hong, J.-W. \& Levine, M. Regulation of Hox gene activity by transcriptional elongation in Drosophila. Curr. Biol. 19, 688-693 (2009).

3. Oh, K. S. et al. Phenotypic heterogeneity in the XPB DNA helicase gene (ERCC3): xeroderma pigmentosum without and with Cockayne syndrome. Hum. Mutat. 27, 1092-1103 (2006).

4. Chopra, V. S. et al. The polycomb group mutant esc leads to augmented levels of paused Pol II in the Drosophila embryo. Mol. Cell. 42, 837-844 (2011).

5. Cleaver, J. E. Cancer in xeroderma pigmentosum and related disorders of DNA repair. Nat. Rev. Cancer 5, 564-573 (2005).

6. Levine, M. Paused RNA polymerase II as a developmental checkpoint. Cell 145, 502-511 (2011).

7. Compe, E. \& Egly, J.-M. TFIIH: when transcription met DNA repair. Nat. Rev. Mol. Cell Biol. 13, 343-354 (2012).

8. Boyle, J. et al. Persistence of repair proteins at unrepaired DNA damage distinguishes diseases with ERCC2 (XPD) mutations: cancer-prone xeroderma pigmentosum vs. non-cancer-prone trichothiodystrophy. Hum. Mutat. 29, 1194-1208 (2008).

9. Bentley, D. L. \& Groudine, M. A block to elongation is largely responsible for decreased transcription of c-myc in differentiated HL60 cells. Nature 321, 702-706 (1986).

10. Strobl, L. J. \& Eick, D. Hold back of RNA polymerase II at the transcription start site mediates down-regulation of c-myc in vivo. EMBO J. 11, 3307-3314 (1992).

11. Trumpp, A. et al. c-Myc regulates mammalian body size by controlling cell number but not cell size. Nature 414, 768-773 (2001).

12. Hultgardh-Nilsson, A., Larsson, S. H., Jin, P., Sejersen, T. \& Ringertz, N. R. Neurokinin A induces expression of the c-fos, c-jun, and c-myc genes in rat smooth muscle cells. Eur. J. Biochem. 194, 527-532 (1990).

13. Eilers, M. \& Eisenman, R. N. Myc's broad reach. Genes Dev. 22, 2755-2766 (2008).

14. Levens, D. 'You Don't Muck with MYC'. Genes Cancer 1, 547-554 (2010). 
15. Dang, C. V. Enigmatic MYC conducts an unfolding systems biology symphony. Genes Cancer 1, 526-531 (2010).

16. Liu, J. et al. The FUSE/FBP/FIR/TFIIH system is a molecular machine programming a pulse of c-myc expression. EMBO J. 25, 2119-2130 (2006).

17. Gallant, P., Shiio, Y., Cheng, P. F., Parkhurst, S. M. \& Eisenman, R. N. Myc and Max homologs in Drosophila. Science 274, 1523-1527 (1996).

18. Johnston, L. A., Prober, D. A., Edgar, B. A., Eisenman, R. N. \& Gallant, P. Drosophila myc regulates cellular growth during development. Cell 98, 779-790 (1999).

19. Schreiber-Agus, N. et al. Drosophila Myc is oncogenic in mammalian cells and plays a role in the diminutive phenotype. Proc. Natl Acad. Sci. USA 94, 1235-1240 (1997).

20. Core, L. J. \& Lis, J. T. Transcription regulation through promoter-proximal pausing of RNA polymerase II. Science 319, 1791-1792 (2008).

21. Benassayag, C. et al. Human $c-M y c$ isoforms differentially regulate cell growth and apoptosis in Drosophila melanogaster. Mol. Cell Biol. 25, 9897-9909 (2005).

22. Boettiger, A. N. \& Levine, M. Synchronous and stochastic patterns of gene activation in the Drosophila embryo. Science 325, 471-473 (2009).

23. Liu, J. et al. The FBP interacting repressor targets TFIIH to inhibit activated transcription. Mol. Cell 5, 331-341 (2000).

24. Liu, J. et al. Defective interplay of activators and repressors with TFIH in xeroderma pigmentosum. Cell 104, 353-363 (2001).

25. Dani, C. et al. Extreme instability of myc mRNA in normal and transformed human cells. Proc. Natl Acad. Sci. USA 81, 7046-7050 (1984).

26. Dani, C. et al. Increased rate of degradation of c-myc mRNA in interferontreated Daudi cells. Proc. Natl Acad. Sci. USA 82, 4896-4899 (1985).

27. Gregory, M. A. \& Hann, S. R. c-Myc proteolysis by the ubiquitin-proteasome pathway: stabilization of c-Myc in Burkitt's lymphoma cells. Mol. Cell Biol. 20, 2423-2435 (2000)

28. Hann, S. R., Thompson, C. B. \& Eisenman, R. N. c-myc oncogene protein synthesis is independent of the cell cycle in human and avian cells. Nature 314, 366-369 (1985)

29. Rabbitts, P. H. et al. Metabolism of c-myc gene products: $c-m y c$ mRNA and protein expression in the cell cycle. EMBO J. 4, 2009-2015 (1985).

30. Sears, R. C. The life cycle of C-myc: from synthesis to degradation. Cell Cycle 3, 1133-1137 (2004)

31. Moberg, K. H., Mukherjee, A., Veraksa, A., Artavanis-Tsakonas, S. \& Hariharan, I. K. The Drosophila F box protein archipelago regulates dMyc protein levels in vivo. Curr. Biol. 14, 965-974 (2004).

32. Li, L., Anderson, S., Secombe, J. \& Eisenman, R. N. The Drosophila ubiquitinspecific protease Puffyeye regulates dMyc-mediated growth. Development 140, 4776-4787 (2013)

33. Chung, H. J. \& Levens, D. c-myc expression: keep the noise down! Mol. Cells 20, 157-166 (2005)

34. Levens, D. \& Gupta, A. Molecular biology. Reliable noise. Science 327, 1088-1089 (2010).

35. Matsushita, K. et al. An essential role of alternative splicing of c-myc suppressor FUSE-binding protein-interacting repressor in carcinogenesis. Cancer Res. 66, 1409-1417 (2006).

36. Rougvie, A. E. \& Lis, J. T. The RNA polymerase II molecule at the 5' end of the uninduced hsp70 gene of D. melanogaster is transcriptionally engaged. Cell 54, 795-804 (1988).

37. Berneburg, M. et al. The cancer-free phenotype in trichothiodystrophy is unrelated to its repair defect. Cancer Res. 60, 431-438 (2000).

38. Weber, A., Liu, J., Collins, I. \& Levens, D. TFIIH operates through an expanded proximal promoter to fine-tune c-myc expression. Mol. Cell Biol. 25, 147-161 (2005).

39. Berneburg, M. \& Lehmann, A. R. Xeroderma pigmentosum and related disorders: defects in DNA repair and transcription. Adv. Genet. 43, 71-102 (2001).

40. Mounkes, L. C., Jones, R. S., Liang, B. C., Gelbart, W. \& Fuller, M. T. A Drosophila model for xeroderma pigmentosum and Cockayne's syndrome: haywire encodes the fly homolog of ERCC3, a human excision repair gene. Cell 71, 925-937 (1992).

41. Mounkes, L. C. \& Fuller, M. T. Molecular characterization of mutant alleles of the DNA repair/basal transcription factor haywire/ERCC3 in Drosophila. Genetics 152, 291-297 (1999).

42. Merino, C., Reynaud, E., Vazquez, M. \& Zurita, M. DNA repair and transcriptional effects of mutations in TFIIH in Drosophila development. Mol. Biol. Cell 13, 3246-3256 (2002).

43. Quinn, L. M. et al. Drosophila Hfp negatively regulates dmyc and stg to inhibit cell proliferation. Development 131, 1411-1423 (2004).

44. Mitchell, N. C. et al. Hfp inhibits Drosophila myc transcription and cell growth in a TFIIH/Hay-dependent manner. Development 137, 2875-2884 (2010).

45. Regan, C. L. \& Fuller, M. T. Interacting genes that affect microtubule function: the nc2 allele of the haywire locus fails to complement mutations in the testis-specific beta-tubulin gene of Drosophila. Genes Dev. 2, 82-92 (1988).
46. Thibault, S. T. et al. A complementary transposon tool kit for Drosophila melanogaster using P and piggyBac. Nat. Genet. 36, 283-287 (2004).

47. Neumann, C. J. \& Cohen, S. M. Distinct mitogenic and cell fate specification functions of wingless in different regions of the wing. Development 122, 1781-1789 (1996).

48. Zecca, M., Basler, K. \& Struhl, G. Sequential organizing activities of engrailed, hedgehog and decapentaplegic in the Drosophila wing. Development 121, 22652278 (1995).

49. Garcia-Bellido, A., Ripoll, P. \& Morata, G. Developmental compartmentalisation of the wing disk of Drosophila. Nat. New Biol. 245, 251-253 (1973).

50. Johnston, L. A. \& Edgar, B. A. Wingless and Notch regulate cell-cycle arrest in the developing Drosophila wing. Nature 394, 82-84 (1998).

51. Herranz, H., Pérez, L., Martín, F. A. \& Milán, M. A Wingless and Notch double-repression mechanism regulates G1-S transition in the Drosophila wing EMBO J. 27, 1633-1645 (2008).

52. Cranna, N. \& Quinn, L. Impact of steroid hormone signals on Drosophila cell cycle during development. Cell Div. 4, 3 (2009).

53. Neto-Silva, R. M., de Beco, S. \& Johnston, L. A. Evidence for a GrowthStabilizing Regulatory Feedback Mechanism between Myc and Yorkie, the\&nbsp;Drosophila Homolog of Yap. Dev. Cell 19, 507-520 (2010).

54. Wu, D. C. \& Johnston, L. A. Control of wing size and proportions by Drosophila myc. Genetics 184, 199-211 (2010).

55. Goodliffe, J. M., Wieschaus, E. \& Cole, M. D. Polycomb mediates Myc autorepression and its transcriptional control of many loci in Drosophila. Genes Dev. 19, 2941-2946 (2005).

56. Penn, L. J. et al. Domains of human c-myc protein required for autosuppression and cooperation with ras oncogenes are overlapping. Mol. Cell. Biol. 10, 4961-4966 (1990).

57. Duman-Scheel, M., Johnston, L. A. \& Du, W. Repression of dMyc expression by Wingless promotes Rbf-induced G1 arrest in the presumptive Drosophila wing margin. Proc. Natl Acad. Sci. USA 101, 3857-3862 (2004).

58. Grewal, S. S., Li, L., Orian, A., Eisenman, R. N. \& Edgar, B. A. Myc-dependent regulation of ribosomal RNA synthesis during Drosophila development. Nat. Cell Biol. 7, 295-302 (2005).

59. Knoblich, J. A. et al. Cyclin E controls S phase progression and its down-regulation during Drosophila embryogenesis is required for the arrest of cell proliferation. Cell 77, 107-120 (1994).

60. Sauer, K., Weigmann, K., Sigrist, S. \& Lehner, C. F. Novel members of the cdc2-related kinase family in Drosophila: cdk4/6, cdk5, PFTAIRE, and PITSLRE kinase. Mol. Biol. Cell 7, 1759-1769 (1996).

61. Datar, S. A., Jacobs, H. W., de la Cruz, A. F., Lehner, C. F. \& Edgar, B. A. The Drosophila cyclin D-Cdk4 complex promotes cellular growth. EMBO J. 19, 4543-4554 (2000).

62. Thacker, S. A., Bonnette, P. C. \& Duronio, R. J. The contribution of E2F-regulated transcription to Drosophila PCNA gene function. Curr. Biol. 13, 53-58 (2003).

63. Komarnitsky, P., Cho, E. J. \& Buratowski, S. Different phosphorylated forms of RNA polymerase II and associated mRNA processing factors during transcription. Genes Dev. 14, 2452-2460 (2000).

64. Fukuda, A., Nogi, Y. \& Hisatake, K. The regulatory role for the ERCC3 helicase of general transcription factor TFIIH during promoter escape in transcriptional activation. Proc. Natl Acad. Sci. USA 99, 1206-1211 (2002).

65. Coin, F., Oksenych, V. \& Egly, J. M. Distinct roles for the XPB/p52 and $\mathrm{XPD} / \mathrm{p} 44$ subcomplexes of TFIIH in damaged DNA opening during nucleotide excision repair. Mol. Cell 26, 245-256 (2007)

66. Andressoo, J.-O. et al. An Xpb mouse model for combined xeroderma pigmentosum and cockayne syndrome reveals progeroid features upon further attenuation of DNA repair. Mol. Cell Biol. 29, 1276-1290 (2009).

67. Van Buskirk, C. \& Schüpbach, T. Half pint regulates alternative splice site selection in Drosophila. Dev. Cell 2, 343-353 (2002).

68. Dietzl, G. et al. A genome-wide transgenic RNAi library for conditional gene inactivation in Drosophila. Nature 448, 151-156 (2007)

69. Huggett, J. F. et al. The digital MIQE guidelines: Minimum Information for Publication of Quantitative Digital PCR Experiments. Clin. Chem. 59, 892-902 (2013).

70. Nelson, J. D., Denisenko, O., Sova, P. \& Bomsztyk, K. Fast chromatin immunoprecipitation assay. Nucleic Acids Res. 34, e2 (2006).

\section{Acknowledgements}

We thank M. Fuller and M. Zurita for the Haywire strains. We are grateful to the Bloomington and VDRC stock centres for Drosophila strains and to the DSHB for antibodies. This work was funded by Project Grants and a Senior Research Fellowship from the National Health and Medical Research Council of Australia. This work was also supported by the Cancer Council of Victoria.

\section{Author contributions}

J.E.A.L., N.C.M., G.P. and L.P. conceived experiments, designed the experiments, performed the experiments, analysed the data and assisted with drafting the manuscript 
O.Z., A.C., and P.M., performed experiments, analysed the data. R.D.H and D.L.L conceived experiments, contributed reagents and assisted with drafting the manuscript. L.M.Q conceived experiments, designed experiments, performed experiments, analysed data and drafted the manuscript.

\section{Additional information}

Supplementary Information accompanies this paper at http://www.nature.com/ naturecommunications
Competing financial interests: The authors declare no competing financial interests.

Reprints and permission information is available online at http://npg.nature.com/ reprintsandpermissions/

How to cite this article: Lee, J.E.A. et al. Defective Hfp-dependent transcriptional repression of dMYC is fundamental to tissue overgrowth in Drosophila XPB models. Nat. Commun. 6:7404 doi: 10.1038/ncomms8404 (2015) 